\title{
How Did It Work? An Examination of the Mechanisms Through Which an Intervention for the Unemployed Promoted Job-Search Behavior ${ }^{1}$
}

\author{
Michelle van Ryn ${ }^{2}$ \\ Department of Health Policy and Management, University at Albany
}

\author{
Amiram D. Vinokur \\ Institute for Social Research, University of Michigan
}

Conducted process analysis of treatment mediation effects (Judd \& Kenney, 1981) on longitudinal data from a large randomized field experiment with 928 recently unemployed persons. The experimental treatment included an intervention that succeeded in promoting quality reemployment outcomes, as described in earlier reports (Caplan et al., 1989; Vinokur et al., 1991). Using Ajzen's theory of planned behavior (Ajzen, 1985, 1988), the analyses examined the mediating effects of job-search self-efficacy, attitude, norms, and intention on job-search behavior. The results provided substantial support for the theory of planned behavior and demonstrated the mediational role of job-search self-efficacy. For both 1-and 4-month posttests, job-search self-efficacy was shown to mediate the effects of the intervention through its direct effects on job-search intention and on short term job-search behavior, as well as through its indirect effects on subjective norms and attitude. However, in the longer term 4-month posttest, exposure to the intervention had a direct effect on job-search behavior. This long-term direct effect of the intervention was hypothesized to reflect the influence of inoculation against setbacks which is essential for sustaining the long-term behavioral efforts involved in job seeking.

\footnotetext{
${ }^{1}$ This article is based on research conducted under National Institute of Mental Health grants 39675 and 2P50MH38330, the latter representing the Michigan Prevention Research Center. This article represents a portion of the doctoral dissertation of Michelle van Ryn. We thank Richard Price and Walter Gregg for their comments on an earlier version of this article. ${ }^{2}$ All correspondence should be addressed to Michelle van Ryn, Department of Health Policy and Management, 203B Husted Hall, University at Albany, Albany, New York 12222.
} 
Loss of a job is a major life event which affects nearly 10 million persons in the United States every year (Riegle, 1982; U.S. Bureau of Labor Statistics, 1992). Even in times of economic growth and prosperity, rates of unemployment remain consistently high in some locations and among some populations (Dooley \& Catalano, 1988). Both cross-sectional and longitudinal studies indicate that unemployment increases the risk of poor mental health in terms of increased depression, anxiety, minor psychiatric morbidity, and decreases self-esteem and life satisfaction (e.g., Cobb \& Kasl, 1977; Dooley \& Catalano, 1980; Kessler, Turner, \& House, 1988; Vinokur, Caplan, \& Williams, 1987; Warr, 1983; Warr, Jackson, \& Banks, 1988). Unemployment also contributes to the deterioration of family and spouse well-being and role functioning (Atkinson, Liem, \& Liem, 1986; Dew, Bromet, \& Schulberg, 1987; Liem \& Liem, 1988) and to lowered subjective perceived competence (Warr et al., 1988), as well as higher suicide and suicide attempt rates (Moser, Fox, \& Jones, 1984; Platt, 1986).

At the same time that longitudinal panel studies document the harmful physical and mental health effects of unemployment, they also provide strong evidence that reemployment restores well-being to levels found among the stably employed (e.g., Caplan, Vinokur, Price, \& van Ryn, 1989; Kessler et al., 1988; Vinokur et al., 1987). Recovery from unemployment depends in large part on successfully securing reemployment. Thus, the primary coping task for the job seeker is conducting an effective job search. However, seeking reemployment is a difficult task. For most of the unemployed, searching for a job demands novel or little known behaviors (Bolles, 1989) performed under stressful conditions (Amundson \& Borgen, 1982). For an intervention to effectively prevent or minimize the negative effects of unemployment it will have to promote persistent and effective job-search behavior.

Prior reports have described the success of one such intervention for the unemployed (Caplan et al., 1989; Price, van Ryn, and Vinokur, 1992; Vinokur, van Ryn, Gramlich, \& Price, 1991). This intervention promoted successful job-search behavior and quality reemployment among an unemployed community sample. The intervention took place within the context of a large $(N=928)$ randomized field experiment which allowed for rigorous tests of experimental main effects, preserving the integrity of the randomized experimental design. These earlier reports demonstrated significant beneficial main effects of the experimental intervention on reemployment rates, quality of reemployment, and pay among the reemployed participants. These main effects were statistically significant using the most conservative test, comparing all those assigned by randomization to the experimental group, including those who did not show up for the intervention, with those respondents assigned to the control group. 
This paper goes beyond these earlier reports in exploring how the intervention produced its beneficial outcomes. Our aim is to investigate the cognitive mediators of the intervention's effects on job-search behavior. In examining the way that the intervention produced beneficial effects, we test the utility of a predictive model that represents an integration of social cognitive theories emphasizing self-efficacy as the primary mediator of behavior change (e.g., Bandura, 1986) with Ajzen and Fishbein's (1980) theory of reasoned action. The theory of reasoned action has been widely applied to the prediction of diverse behaviors with considerable success (see Ajzen \& Fishbein, 1980, for a review), including the prediction of job-search behavior among a community sample of unemployed persons (Vinokur \& Caplan, 1987). The theory of reasoned action places intention to perform the behavior as the sole direct predictor of behavioral performance. In turn, intention to perform a behavior is seen to be entirely predicted by attitude (the perceived likelihood that the behavior will lead to a given outcome $X$ the subjective value placed on the outcome) and subjective norms (the perceived degree to which referent others think the behavior should be performed $X$ the motivation to comply with referent others) regarding the behavior. Ajzen (1985) attempted an integration of behavior-specific self-efficacy beliefs with the theory of reasoned action in his theory of planned behavior. This theory places perceptions of personal control regarding performing a given behavior (conceptually identical to self-efficacy [Ajzen, 1985]) ${ }^{3}$ as an independent determinant of behavioral intention and as a correlate of attitude and subjective norm toward the behavior, which in turn are seen to correlate with each other (Ajzen, 1985, 1991).

The integration of self-efficacy into the theory of reasoned action may be of special interest to those attempting to predict and influence specific coping responses following stressful life events. Research on self-efficacy has become increasingly relevant to understanding both the performance and cost of coping responses. Self-efficacy regarding a specific behavior is defined as the belief that one can successfully execute the behavior or course of action under possibly stressful or novel circumstances (Bandura, 1977, 1986). It can have two interrelated sources of influence on coping responses. First, self-efficacy has been shown to reduce the cost of coping by reducing anxiety and fear associated with a given coping response. For example, experiments performed by Bandura and colleague's provide extensive empirical evidence for the role of perceived self-efficacy in overcoming fear and promoting adaptive coping with threatening tasks. When performing feared tasks (handling snakes, spiders), participants' experimen-

${ }^{3}$ Also confirmed in a personal communication with Ajzen. 
tally manipulated self-efficacy regarding these tasks reduced perceived stress and promoted task performance (Bandura, Reese, \& Adams, 1982; Bandura, Taylor, Williams, Mefford, \& Barchas, 1985). In discussing their findings, Bandura and his colleagues (Bandura, 1986; Ozer \& Bandura, 1990) suggest that self-efficacy serves as a cognitive mechanism through which perceptions of controllability reduce the stress reactions that can occur when individuals are faced with the demand for a given coping response. In this way, self-efficacy may directly reduce the level of effort required to execute a given coping response, as well as the negative impact of the coping demand.

Self-efficacy beliefs regarding a behavior have also been shown to increase the likelihood of performing the behavior, persisting at the behavior, and to improve the quality of performance, thus contributing to desired outcomes. For example, behavior-specific self-efficacy improved athletic performance (Barling \& Abel, 1983; Nelson \& Furst, 1972) math performance and persistence at math tasks (Collins, 1982) and increased the likelihood of desired behavioral outcomes in studies of pain control during labor (Manning \& Wright, 1983). It has also been demonstrated to promote a variety of health behaviors such as smoking cessation and weight loss (for reviews, see Bandura, 1986; O'Leary, 1985) among other.

These studies suggest that self-efficacy regarding a given coping response is a likely determinant of that coping response following stressful events, as well as a determinant of the level of performance. However, few studies test the role of self-efficacy in determining adaptive coping behavior following a major stressful life event among a community sample. The few that exist are largely limited by cross-sectional designs that prevent tests of causality (e.g., Major, Mueller, \& Hildebrant, 1985), or by generalized measures of self-efficacy that may be confounded with self-esteem (e.g., Major et al., 1985; Long, 1989; Mueller \& Major, 1989). Additionally, although empirical tests of Ajzen's expanded model supported the addition of behavioral control as an independent determinant of intention (Ajzen \& Madden, 1986; Schifter \& Ajzen, 1985), these previous tests have been largely limited to small-scale experimental studies with college students. The role of self-efficacy needs to be examined in longitudinal analyses among a community sample. Because this study is based on an intervention that focused on the enhancement of job-search self-efficacy with longitudinal follow-up of an unemployed community sample, it is possible to examine the extent to which self-efficacy causally mediated the effects of the intervention on job-seeking behavior.

The present study examined the role of self-efficacy in mediating intervention effects on job-seeking behavior. Using a longitudinal design, it compared job-search self-efficacy, with job-search attitudes, and subjective 
norms on both their mediating role and on the strength of their effect on job-seeking intention and behavior, representing a test of Ajzen's theory of planned behavior in its addition of self-efficacy to the explanatory variables in the theory of reasoned action. The study tested Bandura's (1977, 1986) assertion that self-efficacy, while not the sole determinant of behavior, is the primary mediator of change in behavior. The strongest determinants of behavioral intention were hypothesized to be attitude and subjective norm, as suggested by the theories of reasoned action (Ajzen \& Fishbein, 1980) and planned behavior (Ajzen, 1985, 1991). The hypothesized predictive model is illustrated in Figure 1, representing Ajzen's theory of planned behavior, and shows the relationship among the variables of interest at pretest. The specific findings are discussed in greater detail later.

\section{METHODS}

\section{Respondents and Procedures}

\section{Recruitment}

Study participants were recruited from four southeast Michigan unemployment compensation offices while waiting on line for unemployment checks. Carefully trained recruiters approached the respondents and asked them whether they would be interested in participating in a University of Michigan job-search skills program which would consist of either a 2-week seminar (experimental condition) or self-instructional materials that participants would receive through the mail (control condition). Respondents who were interested in participating, and who met the other eligibility criteria described below, were asked to sign up for the job-search skills programs as well as an associated study of the experience of unemployment. To prevent a threat to internal validity created by differential disappointment effects, we recruited only those respondents who said they were equally interested in either the seminar or the self-administered program into the randomized experiment. Approximately 35\% of respondents expressed a preference and thus were screened out of the experiment. Of these, $90 \%$ preferred the self-instructional program. All those who were screened out because of having a program preference were sent job-search self-instructional materials. Of the $65 \%$ with no preference we recruited 1,122 who also met the eligibility criteria: They were more than 2 years away from retirement, they did not expect to be recalled to their former jobs, they did not show any obvious signs of mental illness, and they had been unemployed for 4 months or less. 


\section{Characteristics of the Sample}

The sample was intended to represent a broad range of unemployed people but was not intended to be a random sample of the unemployed work force. For example, recruitment from the state employment offices defined the population as persons who were eligible for unemployment compensation and/or decided to apply for such compensation. The obtained sample was similar in some ways to the United States unemployed population over 16 years of age (U.S. Bureau of Labor and Statistics, 1986) and to a representative community survey sample of unemployed (Kessler et al., 1988). Males constituted $46 \%$ of the sample compared to $60 \%$ in the community survey and $56 \%$ in the U.S. population. Blacks constituted $15 \%$ in our study compared to $20 \%$ in the community survey and $22 \%$ in the U.S. population. The average age was 35.9 years $(S D=10.6)$ and the average education was about 12.9 years $(S D=1.9)$. Similarly, the average age in the community survey was 35.0 years $(S D=10.5)$ and the average education was about 12.0 years $(S D=2.4)$, as it is in the U.S. population. Finally, $53 \%$ of our sample respondents included persons who were single at the time, that is, respondents who were divorced, separated, widowed, or never married.

Nearly one third of the sample fell into each of the following three broad occupational classifications: (a) professional and managerial (28\%), (b) service and clerical (33\%), and (c) blue collar (38\%). Using an 8-category code based on the Standard Industrial Classification Index (1972), the most common occupation was clerical (30\%). This group was followed by managers $(16 \%)$, operators $(13 \%)$, craft workers $(11 \%)$, and professionals $(10 \%)$ with the remaining $20 \%$ being miscellaneous.

The participants in this study were, on the average, well into their careers; respondents reported being with their previous employer an average of 6 years $(S D=6.3)$. Average length of unemployment was 9 weeks $(S D=4.5)$.

\section{Administration of Pre- and Posttest Questionnaire Instruments}

All study participants were included in a pretest 2 weeks before the intervention (T1) and posttests 1 (T2) and 4 months (T3) after the intervention. All pretest and posttest measures were collected using self-administered questionnaires that were mailed to the respondents with a $\$ 5$ bill as payment for completing the questionnaire and a prepaid return envelope. The measures were identical in form and administration for all experimental conditions. Of those recruited $83 \%$ responded at pretest, $88 \%$ 
of those who completed the pretest responded at the 1-month posttest, and $80 \%$ of those who completed the pretest responded at the 4-month posttest.

\section{Control and Experimental Groups}

Upon successful recruitment, respondents were randomized into the experimental $(n=752)$ and control $(n=370)$ conditions of the study. Of those assigned to the experimental and the control conditions, $606(81 \%)$ and $322(87 \%)$, respectively, returned the pretest questionnaire and became enrolled in the study. Experimental group respondents were sent an invitation to participate in the job-search skills seminar along with persuasive messages and informational materials. Control group respondents were sent written self-administered job-search materials and a pamphlet on coping with unemployment. There were no significant differences at pretest between the experimental and control groups on demographic variables, jobseeking motivation, mental health, or any other dependent variables. Of those respondents who were randomly assigned to the experimental group and therefore invited to the intervention, 59\% did not show up for the intervention at all. However, these nonattenders (no-shows) completed all of the follow-up questionnaires and their data were included in all the analyses used to evaluate the effectiveness of the intervention as reported in Caplan et al. (1989) as well as in the analyses described below. The remaining $41 \%(n=308)$ of the experimental group who attended the intervention varied in the number of sessions they attended (mean number of sessions $=6.2, S D=2.1$ ).

\section{The Experimental Treatment}

The intervention offered to the experimental group consisted of eight 3 -hour group sessions over a 2-week period. Sessions included between 15 and 20 participants and were facilitated by a cotrainer pair. The ultimate objective of the intervention was to prevent the negative mental health effects of unemployment and to promote quality reemployment. The intervention can be characterized as having five major overlapping components:

Job-Search Skill Training. Participants were invited to acquire and rehearse job-search skills in a safe, supportive environment. They were taught the job-search skills and techniques that job-search specialists recommend most highly, using a learning process based on social learning theory. This component was intended to increase participant job-search self-efficacy, foster a positive attitude toward job-seeking, increase job-search knowledge 
and skills, increase job-search motivation, and reduce feelings of helplessness.

The Learning Process. Participants' knowledge and ideas were elicited through small and large group discussions, brainstorming sessions, and other activities. Participants spent much of their time rehearsing new skills and providing each other with support. This process was intended to maximize the effectiveness of the learning environment and promote participants' self-efficacy, sense of self-worth, and involvement in the job-search process.

Inoculation Against Setbacks. This component was derived from Meichenbaum's (1985) work on individual counseling and Janis and Mann's (Janis, 1982; Janis \& Mann, 1977) work on behavior change and helping individuals adhere to difficult decisions (e.g., weight loss). The intervention emphasized an extension of their work in which the group anticipated possible setbacks or barriers and prepared to overcome them through a problem-solving and skill acquisition process. This component was intended to increase specific coping skills for dealing with the social, emotional, and task demands associated with job loss and the job search.

Social Support from Trainers and Group. Trainers expressed empathy and validation of participants' concerns and feelings, and expressed encouragement for participants' coping efforts. Trainers modeled and reinforced supportive behaviors for participants. Group exercises were designed so as to provide opportunities and reinforcement for participants' supportive behaviors toward each other. This component was intended to provide social support and reduce feelings of isolation.

Trainer Referent Power. Based on Janis and colleagues' (Janis, 1982a, $1982 b$; Janis \& Mann, 1977) work on the bases of social influence of supportive helpers, the supportive influence trainers had with participants was seen to be based on participants' experience of trainers as reliable self-esteem enhancers, as referent persons whom they esteem and desire the esteem of. This required that trainers build trust and reduce social distance by providing participants with unconditional positive regard, specific positive feedback, moderate self-disclosure, and encouragement for participants' self-disclosure.

\section{Measures}

All the constructs were assessed through multi-item indices with the exception of "exposure to the intervention" as described below. Indices 
were developed through standard item-analysis and factor-analysis procedures.

The exposure to intervention measure was based on attendance records kept during the intervention and consisted of the number of intervention sessions attended out of the eight offered. The measure was created according to the guidelines suggested by Judd and Kenney (1981) for performing mediational analyses. Those participants assigned to the control group as well as those who were assigned to the experimental group but failed to show up were assigned a value of 0 on this variable. An additional experimental assignment dummy variable was created for conservative analyses of main effects. Those participants randomized into the experimental group were assigned a 1, while control group respondents were assigned a 0 . The exposure to intervention measure was applied to the mediational analyses presented below, although substitution of the experimental assignment dummy variable yielded the same pattern of results.

The job-search self-efficacy measure consisted of 6 items. All respondents, regardless of reemployment status, were asked to rate how confident they felt about being able to do the following things successfully: make the best impression and get points across in an interview, contact and persuade employers to consider them for the job, complete a good job-application or resume, use friends or other contacts to discover promising job-openings, use friends and other contacts to find out about employers that need their skills, and make a good list of all their skills that can be used to find a job. The ratings on a scale categories ranging from not at all confident (1) to a great deal confident (5) were averaged to create a job-search self-efficacy index. Cronbach's alpha coefficient for this index was .87 (Nunnally, 1978).

Data on attitude, intention, and subjective norm toward job-seeking were collected from all the respondents at pretest and from only those respondents who reported not working enough at the corresponding posttest. These measures were used in an earlier investigation by Vinokur and Caplan (1987). They were originally constructed following guidelines set forth by previous investigations of the theory of reasoned action (Ajzen \& Fishbein, 1980).

Attitude toward job-search behavior was measured by ratings on three 7-point scales ranging from extremely wise (beneficial, useful) to extremely foolish (harmful, useless) to try hard in the next 4 months to get a job. The 3-item index, based on the mean of the ratings, had a reliability alpha coefficient of 86 .

Subjective norms toward job-search behavior were assessed by asking respondents to indicate how hard his or her significant other thought he 
or she should try to get a job in the next 4 months and how hard other people who are important to him or her thought he or she should try to get a job in the next 4 months. These two items combined into an index with a reliability alpha coefficient of .80 .

Intention to engage in job-search behavior was assessed using the questions: "In the next four months, how hard do you intend to try to find a job-where you'd work over 20 hours a week?" and "In the next four months how likely is it that you will try hard to get a job?" The rating scale for the first question ranged from not at all hard (1) to extremely hard (5) and for the second question from extremely likely (1) to extremely unlikely (7). The mean of the two ratings made up an index with a reliability alpha coefficient of .80 .

Job-search behavior was assessed by a 10 -item index. Respondents were asked to indicate the number of times in the past month (at the 1month posttest) or 4 months (at the 4-month posttest) they had engaged in each of the following job-search activities: read the newspaper or other publications for job-opportunities, checked with employment agencies, talked to friends, family, or other people for job leads, used or sent out a resume, filled out application forms, telephones, wrote, or visited potential employers; went on a job interview, did things to improve the impression they made, contacted public employment service, or conducted information interviews. A job-search behavior index was constructed from these items with an internal reliability alpha coefficient of .86 .

\section{RESULTS}

As mentioned above, Caplan et al. (1989) reported the significant beneficial main effects of the intervention on reemployment rates, pay per hour, quality of reemployment, and job-search self-efficacy at 1- and 4month posttests. Using other statistical techniques, Vinokur et al. (1991) also demonstrated the significant effects of the intervention on employment (hours worked per week), earnings, and wage rate. These significant main effects were based on the intact randomized experimental design. To examine the mediators of these intervention effects, a series of analyses were performed based on Judd and Kenny's (1981) guidelines for estimating mediators in treatment evaluations. According to Judd and Kenny, the first condition for demonstrating mediation is establishing that the "treatment" (in this case the experimental intervention) directly affects the outcome 
variable of interest (job-search behavior) ${ }^{4}$ Therefore, we applied multiple regression analyses to explore the overall effect of the experimental treatment on job-search behavior and on the cognitive mediational variables: self-efficacy, attitudes, subjective norms, and intentions. Following Judd and Kenny's (1981) guidelines, those assigned to the control group and those assigned to the experimental group who did not attend (no-shows) are assigned a zero on the variable indicating degree of exposure to the intervention. ${ }^{5}$ However, this type of estimation of experimental effects may include bias due to self-selection into the no-show category. To control for self-selection bias, the regression analyses include the pretest measure of each respective dependent variable, as well as pretest age, education, sex, and income. ${ }^{6}$ Thus, these regression analyses provide estimates of the effects of exposure on each variable independent of pretest level of that variable and socioeconomic status. The results of these analyses are presented in Table $I$.

The results reveal that exposure to the experimental intervention was a significant determinant of job-search behavior at the 1 -month posttest $(\beta$

\footnotetext{
${ }^{4}$ The intervention was designed to achieve its goal of reemployment through the enhancement of job-search behavior. Job-search behavior is an important determinant of reemployment, however the relationship between behavior and reemployment is complex. Reemployment is affected by many personal and structural variables such as the occupational qualifications of the applicant and the demands of the job market, in addition to job-search behavior. Additionally, it is difficult to accurately assess the relationship between job-search behavior over the last month and reemployment at the posttest measurement point because job-search ceases once reemployment is achieved, so a linear (or even monotone) relationship between job search and reemployment may not be found. This is further complicated by the fact that a job offer may cause a respondent to cease looking for a job, yet there may be a gap in time between the job offer and when the respondent starts work. Despite the complicated relationship between these two variables, job-seeking behavior measured at the 1-month posttest was a statistically significant predictor of reemployment (the number of hours employed per week) reported at the 4 -month posttest $(\beta=.09 ; p<.01)$, controlling for the number of hours employed per week reported at the 1 -month posttest.

${ }^{5}$ To provide a more conservative test, we repeated all the mediational analyses substituting a dummy variable representing random assignment to condition for the "exposure to intervention" measure. These analyses consistently reveal the same pattern of results demonstrated in Figures 2 and 3. Figures 2 and 3 show the effect of degree exposure to the intervention, representing dosage.

${ }^{6}$ No-shows vs. shows. Those respondents randomized into the experimental group who failed to attend the intervention differ from those who attended on a number of characteristics at pretest. No-shows are younger (mean age $=33$ years vs. 39 in the show group, $t=6.97, p$ $\leq .001$ ), less educated (mean years of education $=12.7$ vs. 13.2 in the show group, $t=3.5$, $p \leq .0001)$, and report less income in the last year $(M=\$ 21,118$ vs. $\$ 24,999$ in the show group). Additionally they report greater job-search self-efficacy than those who showed up for the intervention ( $M=3.67$ vs. 3.45 for the show group, $t=3.07, p \leq .01$ ). There were no significant pretest differences between the show and no-show groups on sex, any of the other cognitive job-seeking variables, or on overall job-search behavior.
} 
Table I. Beta Weights and Multiple Correlation Coefficients of the Effects of Exposure to the Intervention on Job-Search Self-Efficacy, Attitude, Subjective Norms, Intention and Behavior with Pretest Scores of the Respective Dependent Variables, Age, Education, and Family Income as Controls

\begin{tabular}{lccccc}
\hline \multicolumn{1}{c}{ Predictors } & Exposure & $\begin{array}{c}\text { Pretest score } \\
\text { of dependent } \\
\text { variable }\end{array}$ & $R$ & $F(\mathrm{df})$ \\
\hline $\begin{array}{l}\text { Job-search self-efficacy } \\
\text { 1-Month posttest }\end{array}$ & $.27^{b}$ & $.53^{b}$ & $.57^{b}$ & 75.1 & $(5,787)$ \\
4-Month posttest & $.18^{b}$ & $.46^{b}$ & $.50^{b}$ & 50.9 & $(5,762)$ \\
Job-search attitude & & & & & \\
1-Month posttest & .03 & $.49^{b}$ & $.50^{b}$ & 37.0 & $(5,565)$ \\
4-Month posttest & .01 & $.47^{b}$ & $.47^{b}$ & 19.9 & $(5,352)$ \\
Job-search subjective norm & & & & & \\
1-Month posttest & .03 & $.57^{b}$ & $.60^{b}$ & 57.5 & $(5,516)$ \\
4-Month posttest & -.004 & $.52^{b}$ & $.58^{b}$ & 33.6 & $(5,334)$ \\
Job-search intention & & & & & \\
1-Month posttest & .05 & $.52^{b}$ & $.53^{b}$ & 45.0 & $(5,568)$ \\
4-Month posttest & -.03 & $.46^{b}$ & $.47^{b}$ & 20.2 & $(5,360)$ \\
Job-search behavior & & & & & \\
1-Month posttest & $.08^{a}$ & $.59^{b}$ & $.60^{b}$ & 87.1 & $(5,759)$ \\
4-Month posttest & $.09^{a}$ & $.24^{b}$ & $.30^{b}$ & 14.1 & $(5,739)$ \\
\hline
\end{tabular}

${ }^{a} p \leq .01$
${ }_{p}^{b} \leq .001$

$=.08, p \leq .01)$ and the 4-month posttest $(\beta=.09, p \leq .01)$ independent of pretest levels of this variable and sociodemographic controls. Exposure to the intervention was a determinant of job-search self-efficacy at both the 1-month $(\beta=.27, p \leq .001)$ and 4-month posttests $(\beta=.18, p \leq .001)$, once again independent of pretest levels of this variable and age, family income, and education. Exposure to the intervention had no statistically significant effect on the other possible mediators, that is, job-search attitude, subjective norms, or intention. This suggests that self-efficacy is the sole cognitive mediator of the intervention's effects.

\section{Examining the Cognitive Mediators of the Experimental Intervention's Effects on Job-Search Behavior}

The first series of analyses was performed on the pretest data in order to identify the baseline explanatory model of the relationship among the variables. The next series of analyses involved the replication of the first series with the introduction of the amount of exposure to the intervention 
as the antecedent causal factor in a longitudinal model. Also included in these analyses are the corresponding pretest measures, as well as pretest education, family income, and age as partial controls for self-selection biases (these are the only sociodemographic variables for which there were significant differences between shows and no-shows). The last series included variables measured at three different time periods and thus represents the most comprehensive longitudinal analyses and also includes the pretest controls. Separate analyses were performed on men and women to search for gender differences in the pattern of results. The results of the gender subgroup analyses revealed that the mediators of the intervention and the relationship among these mediators is very similar for both men and women. In the absence of evidence for gender differences, the analyses presented below were performed on the entire sample.

As mentioned above, the first series of path analyses included data collected prior to the intervention and was modeled after Ajzen's theory of planned behavior. These path analyses identify the initial explanatory model for the later analyses that include exposure to the intervention. Specifically, pretest levels of job-search behavior and job-search intention were regressed on pretest levels of job-search self-efficacy, job-search attitude, and job-search subjective norm. Figure 1 displays the path coefficients and multiple correlation coefficients. ${ }^{7}$ The model on Figure 1, as well as all the other models presented, is a reduced path model. All possible paths were tested, however only pathways that achieve statistical significance $(p \leq .05)$ are presented.

The results presented in Figure 1 are consistent with the theory of planned behavior with the exception of one pathway. As hypothesized, jobsearch self-efficacy was shown to be a significant predictor of intention $(\beta$ $=.05, p \leq .05)$, and of job-search behavior $(\beta=.19, p \leq .001)$ independent of subjective norm, attitude, and intention to search for a job. As is consistent with the theory of planned behavior, attitude and subjective norm toward job-seeking are the strongest predictors of behavioral intention $(\beta$ $=.49$ and .35 , respectively; both $p s \leq .001$ ). The results presented in Figure 1 also show a significant association between self-efficacy and attitude $(r$ $=.17, p \leq .01)$ and subjective norm $(r=.13, p<.01)$ toward job-seeking, as well as between subjective norm and attitude $(r=.47, p \leq .001)$. Contrary

\footnotetext{
${ }^{7}$ Multiple correlation coefficients include pretest scores on the dependent variables as controls. However, to preserve the clarity of the figures, the paths representing the effects of the pretest scores on the dependent variables are not presented. For those who prefer to interpret the residuals of the dependent variable instead of the multiple correlation coefficient, the multiple correlation coefficient can be converted by taking the square root of $\left[1-R^{2}\right]$. Thus, the residual for Job Search Behavior in Figure 1 is .91; the residual for Job Search Behavior in Figure 2 is .69; the residual for Job Search Behavior in Figure 3 is .85 .
} 

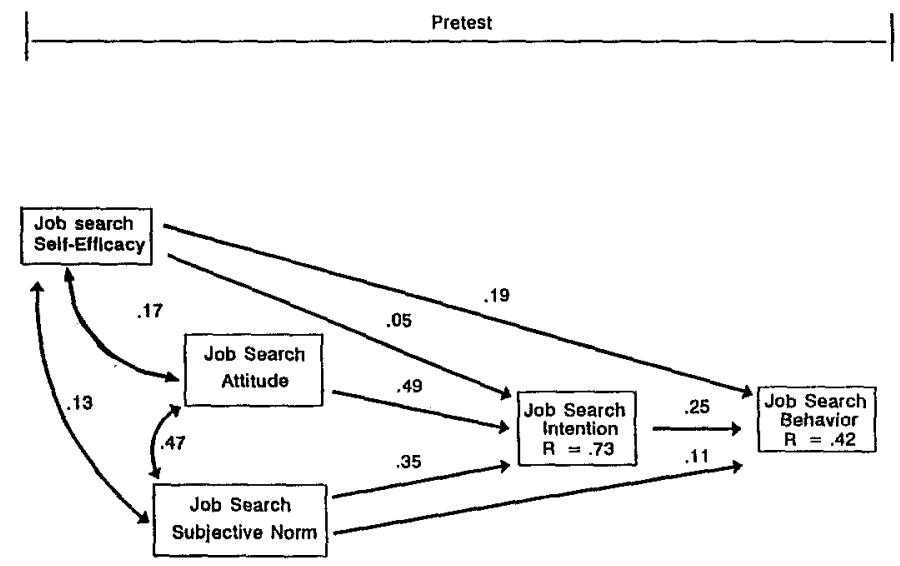

Fig. 1. Path diagram of the determinants of job search behavior among the unemployed sample at pretest $(n=927)$. Only statistically significant paths beyond the .05 level are presented with their coefficients (standardized regression beta weights). The multiple correlation coefficients $(R)$ are statistically significant at the .001 level.

to the theory of planned behavior, subjective norm is a direct predictor of job-search behavior $(\beta=.11, p \leq .01)$, independent of both inattention and self-efficacy.

Figure 2 presents the estimated causal model examining the role of experimentally manipulated self-efficacy in mediating the effect of exposure to the intervention (the number of intervention sessions attended) on jobsearch behavior at the 1-month posttest. Once again, all possible paths were tested but only the statistically significant pathways are included in the model. Pretest scores for age, education, family income (the sociodemographic variables distinguishing shows from no-shows), and sex, as well as pretest scores for each dependent variable were entered into each equation as partial controls for self-selection bias. Finally, the analyses for this model included only those respondents who reported that they were not working enough at the 1 -month posttest $(n=565)$ and therefore were the relevant subsample of persons who were still searching for a job.

Again, the results presented in Figure 2 provide substantial support for the theory of planned behavior. Job-search self-efficacy was a significant predictor of intention $(\beta=.06, p \leq .01)$, independent of pretest levels of intention and the other explanatory variables in the model. Self-efficacy was a direct determinant of job-search behavior independent of intention to perform the behavior $(\beta=.06, p \leq .05)$. As expected, attitude and subjective norm toward job-seeking were the strongest predictors of behavioral intention $(\beta=.47$ and .31 , respectively; both $p$ s $\leq .001)$. 


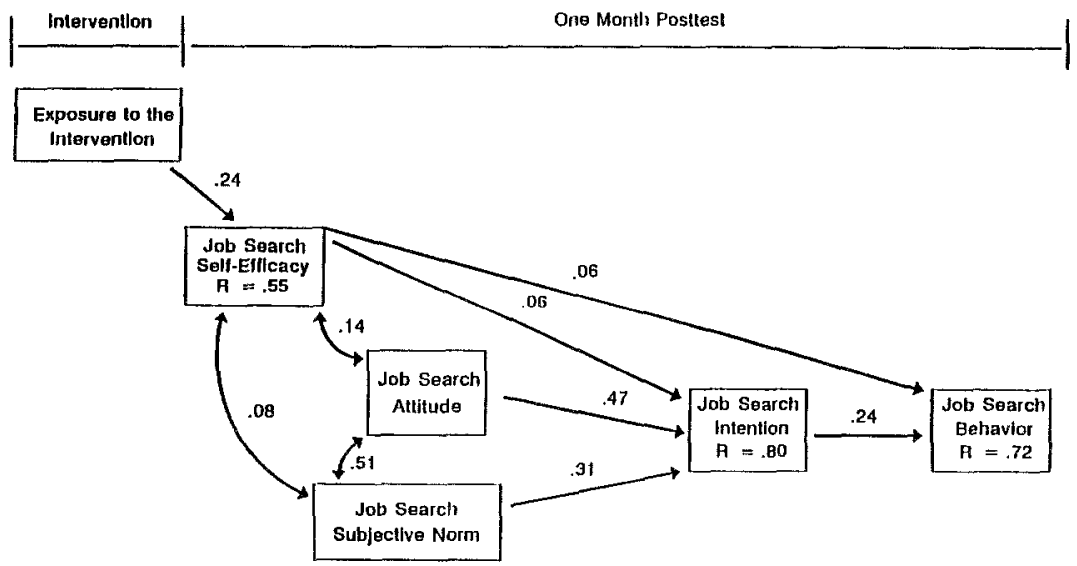

Fig. 2. Path diagram of the mediators of intervention effects on job search behavior among the unemployed sample at the 1-month posttest $(n=565)$. Only statistically significant paths beyond the .05 level are presented with their coefficients. The multiple correlation coefficients are statistically significant at the .001 level. Path coefficients are independent of (controlled for) pretest scores on the dependent variable, age, family income, sex, and education.

The results also reveal that the effects of the experimental intervention on job-seeking behavior were entirely mediated through self-efficacy. Exposure to the intervention had a major effect on job-search self-efficacy $(\beta=.24, p \leq .001)$. Moreover, the intervention had no direct effects on any of the original explanatory variables in the theory of reasoned action (attitude, subjective norm, or intention). Lastly, self-efficacy was a significant correlate of job-search subjective norm $(r=.08, p \leq .05)$ and jobsearch attitude $(r=.14, p \leq .01)$.

Figure 3 presents the results of the mediational analyses examining the role of self-efficacy, measured 1 month after the intervention, in determining job-search behavior exhibited between the 1- and 4-month posttests. This panel design provides a more rigorous test of causality than presented in Figure 2 because it is based on data from four time periods: pretest, intervention, and 1- and 4-month posttests. Once again, pretest scores on the dependent variables as well as age, family income, sex, and education are entered as controls.

The results displayed in Figure 3 replicate the pattern of general support for the theory of planned behavior represented in Figures 1 and 2 . The major determinant of job-search behavior was intention $(\beta=.26, p \leq$ $.001)$, which in turn was determined by a combination of attitude $(\beta=$ $.48)$, subjective norm $(\beta=.32)$, and job-search self-efficacy $(\beta=.06)$, all statistically significant beyond the .05 level. 


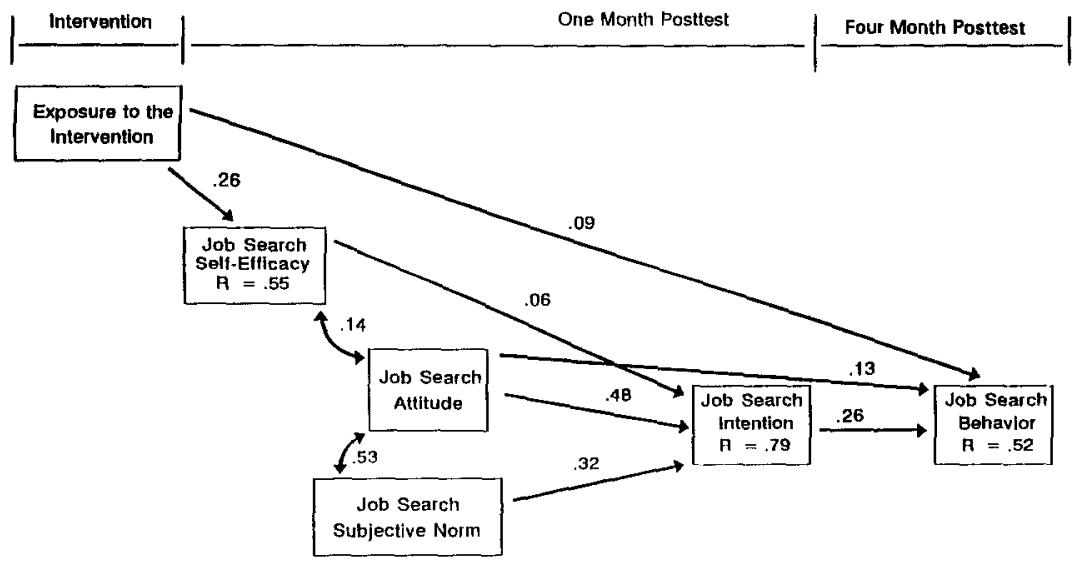

Fig. 3. Path diagram of the mediators of intervention on job search behavior among the unemployed sample at 4 -month posttest $(n=505)$. Only statistically significant paths are presented with their coefficients. The multiple correlation coefficients are statistically significant at the .001 level, Path coefficients are independent of (controlling for) pretest scores on the dependent variable, age, family income, sex, and education.

With regard to the role of self-efficacy, the results confirmed its mediational effects on intention $(\beta=.06, p \leq .05)$. However, in this examination of long-term effects, self-efficacy did not have a direct effect on job-seeking behavior. Unlike the results of the earlier analyses, exposure to the intervention was found here to have a direct influence on job-search behavior $(\beta=.09, p \leq .01) 4$ months later.

\section{DISCUSSION}

Our process analysis of the mediation effects of the intervention followed the methodological guidelines suggested by Judd and Kenny (1981) and the theoretical model of Ajzen as formulated in the theory of planned behavior (Ajzen, 1988). The latter is especially relevant to the investigation because it integrates the role of self-efficacy with an attitude-behavior model, the theory of reasoned action (Ajzen \& Fishbein, 1980).

Our results need to be examined with regard to the potential weaknesses of the study. Attrition is a pervasive problem in most field experiments and it results from two sources. First, participants who initially enroll in the study fail to provide data in subsequent follow-ups, and, second, participants who are randomly assigned to a treatment condition fail to show up for the treatment. In the present investigation, response rates were quite high, $88 \%$ in T2 and $80 \%$ in T3. Most important, analyses of many 
demographic and attitudinal characteristics of the dropouts revealed no interaction effects with experimental conditions.

A second potential problem in our study appears to result from the attrition of those randomly assigned to the experimental condition who did not show up for the intervention. These no-shows differed from the shows in having less education, being younger, and having lower family incomes at pretest. Additionally, they had higher mean job-search self-efficacy at pretest than those who participated in the intervention. There were no differences between shows and no-shows on any other sociodemographic or job-search variables. Despite their failure to show up for the intervention, the no-show respondents continued to provide follow-up data that were included in all the analyses reported earlier (Caplan et al., 1989; Vinokur et al., 1991). As suggested by Cook and Campbell (1979), the main effects of the intervention on reemployment and self-efficacy were demonstrated unequivocally using the original randomized design, which included the noshows in the experimental condition. Furthermore, Table I presents the results of more refined analyses suggested by Judd and Kenny (1981) in which the statistically significant effects of the intervention on both jobsearch self-efficacy and job-search behavior were clearly demonstrated. These analyses were conducted including age, education, family income, and pretest scores on the dependent variables as controls for self-selection bias. Moreover, when we substituted a dummy variable that preserved the original randomized design for the exposure to the intervention variable used in the analyses reported here, we obtained the same pattern of results. Last but not least, and as an added caution, all of our analyses included pretest age, income, sex, and education as well as $\mathrm{T} 1$ pretest measures of the relevant dependent variable as partial controls for self-selection effects. As expected (Fishbein \& Ajzen, 1975), the pattern of results with and without these sociodemographic controls was virtually the same. Additionally, it is important to note that any self-selection bias that remains despite these controls is likely to dampen the observed associations between exposure to the intervention and the job-search self-efficacy. This is because the noshows, coded as 0 exposure to the intervention, reported significantly greater job-search efficacy at pretest than the respondents who attended the intervention.

Another potential weakness in this investigation is the reliance on self-report measures. Self-report measures are the most appropriate ones for assessing attitudes, intention, and perceived self-efficacy. As for jobsearch behavior, it constitutes a behavioral category (Ajzen \& Fishbein, 1980) of many specific behaviors enacted over a long period that cannot be practically observed in a field study. To reduce possible bias, our assessment of this construct included several items, each focusing on a spe- 
cific job-search behavior. Finally, a strong indication for the absence of a generalized positive self-report bias is provided by the fact that only selfefficacy was demonstrated to be increased by the intervention. Other constructs, including attitude, subjective norm, and intention toward job-seeking, were unaffected. The latter manifested the divergent validity (Campbell, 1960) of our results.

The results provided substantial support for the theory of planned behavior and demonstrated the mediational role of job-search self-efficacy. For both 1- and 4-months posttests, job-search self-efficacy was shown to mediate the effects of the intervention through direct effects on intention (and on behavior at the 1-month posttest) and through its indirect effects on subjective norms and attitude. However, in the 4-month posttest, exposure to the intervention had a direct effect on job-search behavior thereby limiting the mediational role of self-efficacy.

Overall support for the theory of planned behavior is provided by the finding that self-efficacy was an independent determinant of behavioral intention in all the causal models presented above. This finding is consistent with those shown in Schifter and Ajzen's (1985) study of weight loss and Meyerowitz and Chaiken's (1987) and Ronis and Kaiser's (1989) study of breast self-examination. Self-efficacy generates expectancies that one can perform the behavior successfully, which in turn are likely to increase the intention to perform the behavior. Conversely, having little confidence in one's ability to execute a behavior is likely to undermine the intention regardless of attitudes or subjective norms toward the behavior.

The results demonstrate that at the 1-month posttest, self-efficacy was the sole mediator of the intervention effects and an independent determinant of behavior. However, at the 4-month posttest, the entire effect of job-search self-efficacy on long-term job-search behavior was mediated through attitude, subjective norm, and behavioral intention. This decay in the effect of postintervention self-efficacy on behavior performed a long time thereafter is not surprising. Weinberg, Gould, Yukelson, and Jackson (1981) found that self-efficacy predicted initial athletic performance, but each additional experience with the task prompted reappraisals of self-efficacy such that the updated perceptions had the major effect on performance. It is quite possible that in the 3 months between posttests, the tumultuous nature of the job-search influenced job-search self-efficacy such that only more proximal self-efficacy had an independent effect.

Finally, it is important to note that contrary to the theory of planned behavior, in the 4-month posttest, exposure to the intervention had a direct effect on job-search behavior that was not mediated by any of the cognitive variables in the model. It is hypothesized that this direct effect may be due to other unmeasured intervening variables of the intervention that served 
to maintain persistence over the long haul but did not operate as strongly in the 1-month posttest. In particular, since the intervention included training in inoculation against setbacks, it is reasonable to consider this factor as the cause of the direct effect of the intervention on job-search behavior. For the short run, this factor may not be very influential because optimism is high and setback experiences are few in number. Only in the long run is inoculation against setback essential for sustaining persistence in jobsearch behavior in the face of accumulating disappointments. Obviously this hypothesis could be tested with specially designed measures of inoculation against setbacks which were not available in our study.

In summary, the results demonstrate the primary role of self-efficacy as the sole cognitive mediator of intervention effects on job-seeking as an adaptive coping behavior following the stressful life events of job loss and unemployment. The results show that job-search self-efficacy had relatively durable effects on intention to job-seek over time, despite repeated failures to get a job. This suggests that self-efficacy enhancement is an especially promising intervention tool in cases where successful adaptation requires persistence at coping behaviors over an extended period of time and in spite of rejections or setbacks.

\section{REFERENCES}

Ajzen, I. (1985). From intentions to actions: A theory of planned behavior. In J. Kuhl \& J. Beckman (Eds.), Action-control: From cognition to behavior. Heidelberg: Springer.

Ajzen, I. (1988). Attitudes, personality and behavior. Chicago: Dorsey.

Ajzen, I. (1991). The theory of planned behavior. Organizational Behavior and Human Decision Processes, 50, 179-211.

Ajzen, L., \& Fishbein, M. (1980). Understanding attitudes and predicting social behavior. Englewood Cliffs, NJ: Prentice-Hall.

Ajzen, I., \& Madden, T. (1986). Prediction of goal directed behavior: Attitudes, Intentions, and Perceived behavioral control. Journal of Experimental Social Psychology, 22, 453-474.

Amundson, N., \& Borgen, W. (1982). The dynamics of unemployment: Job loss and job search. Personnel and Guidance Journal, 60, 562-564.

Atkinson, T., Liem, R., \& Liem, J. (1986). The social costs of unemployment: Implications for social support. Journal of Health and Social Behavior, 27, 317-331.

Averill, J. R. (1973). Personal control over aversive stimuli and its relationship to stress. Psychological Bulletin, 80, 286-303.

Bandura, A. (1977). Self-efficacy: Toward a unifying theory of behavior change. Psychological Review, 84, 191-215.

Bandura, A. (1986). Social foundations of thought and action. Englewood Cliffs, NJ: Prentice-Hall.

Bandura, A., Reese, L., \& Adams, N. (1982). Micro analysis of action and fear arousal as a function of differential perceived self-efficacy. Journal of Personality and Social Psychology, $43,5-21$.

Bandura, A., Taylor, C. B., Williams, L., Mefford, I., \& Barchas, J. (1985). Catecholamine secretion as a function of perceived coping efficacy. Journal of Consulting and Clinical Psychology, 53, 406-414. 
Barling, J., \& Abel, M. (1983). Self-efficacy beliefs and tennis performance. Cognitive Therapy and Research, 7, 265-272.

Bolles, R. N. (1989). What color is your parachute? Berkeley, CA: Ten Speed.

Campbell, D. T. (1960). Recommendations for APA test standards regarding construct, trait, and discriminant validity. American Psychologist, 15, 546-553.

Caplan, R. D., Vinokur, A. D., Price, R. H., \& van Ryn, M. (1989). Job seeking, reemployment, and mental health: A randomized field experiment in coping with job loss. Journal of Applied Psychology, 74, 759-769.

Cobb, S., \& Kasl, S. V. (1977). Termination: The consequences of job loss (NIOSH Research Report, DHEW Publication No. 77-224. Washington, DC: U.S. Government Printing Office.

Cohen, S., Evans, G., Stokols, D., \& Krantz, D. (1986). Behavior, health, and environmental stress. New York: Plenum Press.

Collins, J. L. (1982, March). Self-efficacy and ability in achievement behavior. Paper presented at the annual meeting of the American Educational Research Association, New York.

Cook, T. D., \& Campbell, D. T. (1979). Quasi-experimentation: Design \& analysis issues for field settings. Boston: Houghton Mifflin.

Dew, M. A., Bromet, E. J., \& Schulberg, H. C. (1987). A comparative analysis of two community stressors' long term, mental health effects. American Journal of Community Psychology, 15, 167-187.

Dooley, D., \& Catalano, R. (1980). Economic change as a cause of behavioral disorder. Psychological Bulletin, 87, 450-468.

Dooley, D., \& Catalano, R. (1988). Recent research on the psychological effects of unemployment. Journal of Social Issues, 44, 1-12.

Fishbein, M., \& Ajzen, I. (1975). Belief, attitude, intention, and behavior: An introduction to theory and research. Reading, MA: Addison-Wesley.

Janis, I. (1982a). Short Term Counselling: Guidelines based on recent research. New Haven: Yale University Press.

Janis, I. L. (Ed.). (1982b). Counselling on personal decisions: Theory and research on short-term helping relationships. New Haven: Yale University Press.

Janis, I. L., \& Mann, L. (1977). Decision making. A psychological analysis of conflict, choice, and commitment. New York: Free Press.

Judd, C., \& Kenney, D. (1981). Process analysis: Estimating mediation in treatment evaluations. Evaluation Review, 5, 602-619.

Kessler, R. C., Turner, J. B., \& House, J. S. (1988). The effects of unemployment on health in a community survey: Main, modifying, and mediating effects. Journal of Social Issues, $44,69-85$.

Liem, R., \& Liem, J. H. (1988). The psychological effects of unemployment on workers and their families. Journal of Social Issues, 44, 87-105.

Long, B. C. (1989). Sex-role orientation, coping strategies, and self-efficacy of women in traditional and nontraditional occupations. Psychology of Women, 13, 307-324.

Major, B., Mueller, P., \& Hildebrant, K. (1985). Attributions, expectations and coping with abortion. Joumal of Personality and Social Psychology, 48, 585-599.

Manning, M. M., \& Wright, T. L. (1983). Self-efficacy expectancies, outcome expectancies, and the persistence of pain control in childbirth. Journal of Personality and Social Psychology, 45, 421-431.

Meichenbaum, D. (1985). Stress inoculation training: A clinical guidebook. New York: Pergamon.

Meyerowitz, B. E., \& Chaiken, S. (1987). The effect of message framing on breast self-examination attitudes, intention, and behavior. Journal of Personality and Social Psychology, 52, 500-510.

Moser, K., Fox, A. J., \& Jones, P. R. (1984). Unemployment and mortality in the OPCS longitudinal study. Lancet, $8,1324-1328$.

Mueller, P., \& Major, B. (1989). Self-blame, self-efficacy, and adjustment to abortion. Journal of Personality and Social Psychology, 57, 1059-1068. 
Nelson, L. R., \& Furst, M. L. (1972). An objective study of the effects of expectation on competitive performance. Journal of Psychology, 81, 69-72.

Nunnally, J. C. (1978). Psychometric theory. New York: McGraw-Hill.

Office of Management and Budget (1972). Standard Industrial Classification Manual. Washington, DC: Statistical Policy Division.

O'Leary, A. (1985). Self-efficacy and health. Behavioral Research and Therapy, 23, 427-451.

Ozer, E. M., \& Bandura, A. (1990). Mechanisms governing empowerment effects: A self-efficacy analysis. Journal of Personality and Social Psychology, 58, 472-486.

Platt, S. (1986). Suicidal behavior and unemployment: A literature review. In G. Wescott, P. G. Svensson, \& H. F. K. Zollner (Eds.), Health policy implications of unemployment (pp. 87-132). Copenhagen: World Health Organization.

Price, R. H., van Ryn, M., \& Vinokur, A. (1992). Impact of a preventive job search intervention on the likelihood of depression among the unemployed. Journal of Health and Social Behavior, 33, 158-167.

Riegle, D. W., Jr. (1982). The psychological and social effects of unemployment. American Psychologist, 37, 1113-1115.

Ronis, D., \& Kaiser, (1989). Correlates of Breast self-examination in a sample of college women: Analyses of linear structural relations. Journal of Applied Social Psychology, 19, 1068-1084.

Schifter, D., \& Ajzen, I. (1985). Intention, perceived control and weight loss: An application of the theory of planned behavior. Journal of Personality and Social Psychology, 49, 843-851.

U.S. Bureau of Labor Statistics. (1986, July). Current labor statistics: Employment data. Monthly labor review. Washington DC: Author.

U.S. Bureau of Labor Statistics. (1992, June). Current labor statistics: Employment data. Monthly labor review. Washington, DC: Author.

Vinokur, A., \& Caplan, R. D. (1987). Attitudes and social support: Determinants of job-seeking behavior and well-being among the unemployed. Journal of Applied Social Psychology, 17, 1007-1024.

Vinokur, A., Caplan, R. D., \& Williams, C. C. (1987). Effects of recent and past stress on mental health: Coping with unemployment among Vietnam veterans and non-veterans. Journal of Applied Social Psychology, 17, 708-728.

Vinokur, A. D., van Ryn, M., Gramlich, E., \& Price, R. H. (1991). A long term follow-up and cost-benefit analysis of a successful preventive intervention for the unemployed. Journal of Applied Psychology, 76, 1-7.

Warr, P. B. (1983). Job loss, unemployment and psychological well-being. In V. Allen \& E. van de Vliert (Eds.), Role transitions. New York: Plenum Press.

Warr, P. B., Jackson, P., \& Banks, M. (1988). Unemployment and mental health: Sorne British studies. Journal of Social Issues, 44, 47-68.

Weinberg, R. S., Gould, D. Yukelson, D., \& Jackson, A. (1981). The effect of pre-existing and manipulated self-efficacy on a competitive muscular endurance task. Journal of Sport Psychology, 4, 345-354. 